\title{
Prenatal Stress and Mixed-Handedness
}

\author{
BARBARA M. GUTTELING, CAROLINA DE WEERTH, AND JAN K. BUITELAAR
}

\author{
Department of Psychiatry [B.M.G., J.K.B.], Radboud University Medical Center St. Radboud, PO Box 9101, 6500 HB, Nijmegen, \\ The Netherlands; Department of Developmental Psychology [C.W.], Behavioral Science Institute, Radboud University Nijmegen, PO Box \\ 9104, 6500 HE, Nijmegen, The Netherlands
}

\begin{abstract}
Atypical lateralization, as indicated by mixedhandedness, has been related to diverse psychopathologies. Maternal prenatal stress has recently been associated with mixed-handedness in the offspring. In the present study, this relationship was investigated further in a prospective, methodologically comprehensive manner. Stress levels were determined three times during pregnancy by means of questionnaires and measurements of cortisol levels. The handedness of 110 6-y-old children (48 boys) was determined by independent observers. Mixed handedness was defined as using the opposite hand for one or more of the tested activities. Logistic regression analysis showed that more maternal daily hassles in late pregnancy and maternal mixed-handedness increased the chance of mixed-handedness in the offspring. In contrast, more pregnancyrelated fear in late pregnancy and a longer duration of gestation were associated with a smaller chance of being mixed-handed. Prenatal stress measured during the first two periods of pregnancy or determined by cortisol was not related to mixed-handedness in the offspring. In conclusion, reported and physiologic measures of prenatal stress in a moderately stressed pregnant population were only partly related to offspring mixed-handedness. (Pediatr Res 62: 586-590, 2007)
\end{abstract}

A ccording to the fetal programming hypothesis, the environment in utero can alter the development of the fetus during particular sensitive periods, with a permanent effect on the set point of physiologic systems and the phenotype in later years (1).

Maternal prenatal stress has been shown to affect postnatal physical, behavioral, emotional, temperamental, and physiologic outcome of the offspring (2). In animals, maternal prenatal stress has also been linked with atypical laterality in the offspring (3-5). In humans, reduced brain asymmetry and less right-handedness have been proposed as indicators of disturbances in normal brain development and to be related to psychopathology (6). Atypical lateralization, as indicated by mixed-handedness, has been associated with autism (7), dyslexia (8), attention-deficit/hyperactivity disorder (9), and schizophrenia (10).

Two recent studies investigated the possible association between maternal prenatal distress (11) and anxiety (12) and offspring mixed-handedness. Obel and colleagues (11) reported that the 3-y-old children of mothers who had experi-

Received December 12, 2006; accepted June 17, 2007.

Correspondence: Barbara M. Gutteling, Ph.D., Department of Psychiatry, University Medical Center St. Radboud, PO Box 9101, 6500 HB, Nijmegen, The Netherlands; e-mail: B.Gutteling@psy.umcn.nl

This study was supported by a grant from the Van der Gaag Stichting and the Praeventie Fonds (28-2685). enced more distress and stressful life events during the third trimester of pregnancy had a three- to fourfold higher prevalence of mixed-handedness. Glover et al. (12) reported a significant association between prenatal anxiety measured at $18 \mathrm{wk}$ of pregnancy and mixed-handedness of the 42-mo-old offspring. Furthermore, the authors found a positive relationship between maternal mixed-handedness and offspring mixed-handedness.

The two studies measured handedness through parental reports, and although both found maternal prenatal stress to be associated with mixed-handedness, their results differed with respect to the period in pregnancy in which stress exerted this effect.

The goal of the present study was to investigate this subject further by using independent behavioral observations instead of parental report to determine the offspring's handedness. Observations of the child's handedness were expected to be more objective than asking a parent which hand the child used to perform certain actions. Furthermore, prenatal stress was measured in early, middle, and late pregnancy and by both self-report and saliva levels of the stress hormone cortisol. This makes the present study methodologically more comprehensive than earlier studies on the subject.

\section{METHODS}

The present study is part of an ongoing longitudinal prospective investigation of the influence of prenatal factors on fetal behavior and postnatal development. The study started in 1996. Subjects were recruited from a population of pregnant women of the Outpatient Clinic of the Department of Obstetrics of the University Medical Center Utrecht (UMCU) in Utrecht, The Netherlands, between January 1996 and July 1998.

Participation was on a voluntary basis. Written informed consent was obtained from the mothers and verbal informed consent from the children. The UMCU's ethical committee for experiments on human beings approved the study.

The children participating in this study had been followed since their mothers' pregnancy as part of an ongoing prospective longitudinal study of the effects of prenatal stress in mothers on the cognitive and behavioral development of their children (13-19).

With the exception of two boys who did not want to participate in this part of the study, the study group was the same as described in Gutteling et al. (16) (for more details, please refer to that article).

Observational data were collected from 110 (48 boys and 62 girls) children. The mean age of the participating children was $6.7 \mathrm{y}(\mathrm{SD}=0.7 \mathrm{y})$ and ranged from $5.4 \mathrm{y}$ to $8.0 \mathrm{y}$. Of the 110 mothers, 98 completed the questionnaire on handedness, as did 92 fathers. Reasons for not completing

Abbreviations: HPA, hypothalamic-pituitary-adrenal; STAI, State-Trait Anxiety Inventory 
the questionnaire were lack of interest and lack of time. Furthermore, some fathers were not living with their children and had therefore not received the questionnaire.

Psychosocial measures. Psychosocial stress was assessed with five questionnaires. First, the frequency of daily hassles was measured with the Everyday Problem List (20). This self-report questionnaire was used to assess the number of daily hassles during the past 2 mo. Mothers completed the list by marking yes or no on 49 items that were very diverse in nature (e.g. "You had to wait long for an appointment").

The second measure was the impact score on the Life Events Questionnaire (21). The Life Events Questionnaire was used to measure the negative emotionality or impact of the subjects' life events (such as a divorce or moving) of the past year (at 15-17 wk of gestation) or the past 2 months (at $27-28 \mathrm{wk}$ of gestation and at 38-39 wk of gestation). The impact score of this questionnaire is based on the Social Readjustment Rating Questionnaire (22). The higher the impact score, the more life event-related negative emotionality had been experienced by the subject.

Pregnancy-related anxieties were assessed as indicators of psychosocial prenatal stress. These were determined by a Dutch version of the Pregnancy Related Anxiety Questionnaire-Revised (PRAQ) (23). This self-report questionnaire consists of 34 items and has three subscales. In this study sample, only two subscales were used in this study: fear of giving birth (three items) and fear of bearing a mentally or physically handicapped child (four items). Questions concern specific fears and worries related to pregnancy. The Dutch version of the Perceived Stress Scale (PSS) of Cohen and Williamson (24) was used to measure perceived stress. The PSS measures the amount of stress subjects experienced during the past month, on a 4-point Likert scale varying from never to always (e.g. "how often have you felt nervous and "stressed'?").

Finally, the State-Trait Anxiety Inventory (STAI) (25) measured general anxiety in two ways, namely, state anxiety, which is conceptualized as a transitory emotional anxiety, and trait anxiety, which refers to a relatively stable proneness to anxiety. The STAI-state contains 20 statements that ask the respondent how she feels at the moment (e.g. "I feel upset"). Individuals respond to each item on a 4-point Likert scale, indicating the frequency with which each strategy is used (not at all to very much so). The STAI-trait contains 20 statements that ask the respondent how she generally feels (e.g. "I am a steady person"), and which are also answered on 4-point Likert scales ("almost never" to "almost always").

Endocrinological measures. Maternal salivary cortisol levels were determined as a measure of hypothalamic-pituitary-adrenal (HPA) axis function. Samples were collected with Salivettes (Sarstedt Inc.) in each of the three periods of pregnancy on a preselected day every $2 \mathrm{~h}$ between 0800 and $2000 \mathrm{~h}$ to obtain day curves. Samples were stored at $-20^{\circ} \mathrm{C}$ until analysis. The cortisol concentrations were measured without extraction, using an in-house competitive radioimmunoassay with polyclonal anticortisol-antibody (K7348). [1,2-3H(N)]-Hydrocortisone (NET 185, NEN-DUPONT, Dreiech, Germany) was used as a tracer after chromatographic verification of its purity. The lower limit of detection was $0.5 \mathrm{nmol} / \mathrm{L}$ and interassay variation was $11.0 \%, 8.2 \%$, and $7.6 \%$ at $4.7 ; 9.7$, and $14.0 \mathrm{nmol} / \mathrm{L}$, respectively $(n=20)$. More details on the collection of the saliva samples are described in earlier reports on this longitudinal study $(17,18)$.

Because earlier results in this longitudinal project showed associations between prenatal morning cortisol and postnatal child characteristics $(14,15,17)$, the 0800 -h values of each pregnancy period were used as independent variables. Additionally, the mean values, area under curve, and slopes of the cortisol curves for each pregnancy period were included as independent variables in an exploratory fashion. SPSS version 12.1 was used to calculate the slope that consisted of the seven diurnal measurements. It was calculated as follows: we performed general linear modeling using the mothers as the random factor, the slope as the dependent factor, and the seven cortisol variables $(0800 \mathrm{~h}$ to $2000 \mathrm{~h})$ as the covariates. The beta values of the main effects were considered the slope. This slope in cortisol levels throughout the day is related to the shape of the circadian curve and to recovery from daily stress. The area under the curve was determined as follows: (0800-h measure + 1000-h measure $)+(1000-\mathrm{h}$ measure $+1200-\mathrm{h}$ measure $)+$ (1200-h measure + 1400-h measure $)+(1400-\mathrm{h}$ measure $+1600-\mathrm{h}$ measure $)+(1600-\mathrm{h}$ measure $+1800-\mathrm{h}$ measure $)+(1800-\mathrm{h}$ measure + 2000-h measure).

Child handedness. The children were visited at home. During the visit, the children's handedness was determined. Besides the handedness determination, two cognitive tests were performed as well (16). Trained research assistants who were blind to information about maternal prenatal stress evaluated the handedness of the child by scoring which hand the child used to show how they would perform eight daily activities.

The children were sitting on a chair behind a desk while they pretended to perform the activities. Scores were right, left, or both hands. With one exception (see below), the eight activities were those used by Obel and colleagues (11): drawing, buttering bread, holding a toothbrush, using scissors, holding a fork or a spoon, holding an ice cream, taking sweets from a bag, and throwing a ball. We replaced Obel and colleagues' activity of taking sweets from a bag with taking a balloon from a bag. Children actually performed this action because the balloon was a gift that was presented in the body midline. All activities were performed once.

In the present study, mixed-handedness was scored, in line with the definition of Obel et al. (11), when the child performed at least one activity with the opposite hand to that with which the child performed the remaining activities. If a child performed an activity with both hands simultaneously, this activity was not used for determining that child's handedness.

To check the reliability of the handedness scoring, the first author rescored the handedness of 11 of the children from videotapes $(10 \%$ of the study population, chosen at random). The interrater reliability was $100 \%$.

Parent handedness. The handedness of both of the biologic parents was determined with an 11-item Dutch questionnaire that the parents completed shortly after the home visit. Nine items were identical to those on the scale used by Glover et al. (12): with which hand do you write?, draw?, throw a ball?, hold a racket?, clean your teeth?, cut with a knife?, use a hammer?, strike a match?, and use an eraser? We replaced the two remaining items of the Glover et al. questionnaire (deal cards and thread a needle) with stir with a spoon and hold a bottle opener. When the parents used the same hand for the 11 activities, they were accordingly classified as decisively right- or lefthanded. If the parents reported preferring to use the opposite hand for any of the activities, they were classified as mixed-handed.

Covariates. Several possibly relevant variables were included as covariates. Maternal smoking and use of alcohol were measured by self-report during the first period of pregnancy and scored as $0-1$ variables. Seventeen mothers had smoked during pregnancy, and 19 had consumed alcohol. Social economic status was defined by the mother's educational level: low educational level (primary school, $n=4$ ), middle educational level (high school, $n=60$ ), and high educational level (college or academic education, $n=46$ ). Birth weight (mean $=3393 \mathrm{~g}, \mathrm{SD}=496)$, gestational age (mean $=40.1 \mathrm{wk}$, $\mathrm{SD}=1.1 \mathrm{wk})$, maternal age $($ mean $=32.26, \mathrm{SD}=5.03 \mathrm{y})$, maternal mixed-handedness, paternal mixed-handedness, and both parents being mixed-handed, and the child's sex were also included as covariates in the analyses.

Missing values. About $10 \%$ of the prenatal stress data, which were measured three times during pregnancy, were missing. For each particular variable at each particular assessment moment, the missing values were replaced with the mean score of the group as a whole. Although the imputation with group means smoothes the data by reducing differences between persons, it was preferred over other alternatives such as imputation with the subject's score on the missing variable at another assessment moment because the stress variables were not stable throughout pregnancy (i.e. increased or decreased over time) (Table 1).

Fisher exact tests for each variable showed no significant difference in the number of missing values between the mothers of mixed-handed and nonmixed-handed children.

Statistical analyses. Logistic regression analyses were used to analyze the data; these analyses provide the opportunity to correct for possible confounders. All the covariates mentioned above were put into the analyses together with the prenatal stress factors. To investigate whether mixed-handedness could be predicted from the two different types of prenatal stress variables, logistic regressions with stepwise analyses, with computer-derived steps were carried out separately for the maternal psychosocial stress factors and for the maternal cortisol data. With all tests, $p$ values $<0.05$ were considered statistically significant.

\section{RESULTS}

Mixed-handedness. Eighty-three children, five of whom were left-handed, did not use the opposite hand for any of the eight activities ( $75.5 \%$ of the sample), whereas 27 children, four of whom were mainly left-handed, were classified as mixed-handed ( $24.5 \%$ of the sample).

Fifteen children used the opposite hand for one activity (13.6\% of the sample), and one of them was mainly lefthanded (i.e. used the left hand for the remaining activities). Six children used the opposite hand for two activities (5.5\% of the sample); none of them were mainly left-handed. Six children used the other hand for three activities $(5.5 \%$ of the 
Table 1. Descriptives

\begin{tabular}{|c|c|c|c|c|c|c|c|c|c|c|c|c|}
\hline & \multicolumn{4}{|c|}{ First period } & \multicolumn{4}{|c|}{ Second period } & \multicolumn{4}{|c|}{ Third period } \\
\hline & \multicolumn{2}{|c|}{ MH } & \multicolumn{2}{|c|}{$\mathrm{NMH}$} & \multicolumn{2}{|c|}{$\mathrm{MH}$} & \multicolumn{2}{|c|}{$\mathrm{NMH}$} & \multicolumn{2}{|c|}{$\mathrm{MH}$} & \multicolumn{2}{|c|}{$\mathrm{NMH}$} \\
\hline & $n$ & Mean & $n$ & Mean & $n$ & Mean & $n$ & Mean & $n$ & Mean & $n$ & Mean \\
\hline \multicolumn{13}{|l|}{ Psychosocial variables } \\
\hline Daily hassles & 26 & 9.58 & 77 & 10.04 & 26 & 8.19 & 83 & 8.28 & 23 & 7.30 & 78 & 6.86 \\
\hline Perceived stress & 26 & 28.31 & 82 & 27.89 & 26 & 27.52 & 83 & 28.17 & 23 & 27.52 & 78 & 27.36 \\
\hline Fear of giving birth & 19 & 5.32 & 64 & 5.64 & 19 & 5.00 & 63 & 5.59 & 19 & 4.68 & 64 & 5.83 \\
\hline $\begin{array}{l}\text { Fear of bearing a } \\
\text { handicapped child }\end{array}$ & 19 & 8.42 & 64 & 9.39 & 19 & 7.37 & 63 & 8.57 & 19 & 7.12 & 63 & 8.67 \\
\hline State anxiety & 26 & 33.88 & 83 & 32.50 & 26 & 30.77 & 84 & 31.26 & 21 & 31.62 & 79 & 29.97 \\
\hline Trait anxiety & 26 & 34.00 & 81 & 35.26 & 26 & 31.73 & 79 & 34.37 & 23 & 33.04 & 78 & 33.55 \\
\hline \multicolumn{13}{|l|}{ Cortisol variables } \\
\hline Early morning cortisol & 19 & 18.74 & 69 & 20.79 & 20 & 24.30 & 64 & 23.76 & 12 & 23.42 & 48 & 24.12 \\
\hline Mean cortisol & 18 & 10.28 & 59 & 10.65 & 16 & 15.33 & 57 & 14.31 & 10 & 17.30 & 32 & 16.72 \\
\hline Cortisol slope & 14 & -2.49 & 65 & -2.48 & 16 & -1.66 & 58 & -1.42 & 12 & -4.02 & 42 & -4.31 \\
\hline AUC & 18 & 120.17 & 59 & 123.44 & 16 & 180.73 & 57 & 168.32 & 10 & 207.58 & 32 & 199.55 \\
\hline
\end{tabular}

MH, mixed handedness; NMH, nonmixed handedness; AUC, area under the curve.

sample), and three of them were mainly left-handed. One of these children was a girl who used her right hand for three activities, her left for three other activities, and both hands for the remaining two activities.

Fisher exact tests showed that there were no significant differences in which specific activities were responsible for children being classified as mixed-handed.

Gender differences in mixed-handedness were not found: $29.6 \%$ of the boys were $(n=14)$ and $21 \%$ of the girls $(n=$ 13) were mixed-handed (Fisher exact test, $p=0.08$ ). Of the parents, $14.3 \%$ of the mothers $(n=14)$ and $22.8 \%$ of the fathers $(n=21)$ were mixed-handed (not significantly different, Fisher exact test, $p=0.73$ ). The proportion of mixedhanded children in our study was comparable with that reported in other studies: $24.5 \%$ versus $18.4 \%$ in the study of Glover et al. (12) and 8.2\%-35\%, depending on the definition, in the study of Annett (26). Obel and colleagues (11) reported a lower proportion of mixed-handedness $(6.2 \%)$.

Relationship between maternal prenatal psychosocial stress and endocrinological stress. Descriptives of the psychosocial and endocrinological stress scores are given in Table 1.

Correlations (Spearman or Pearson, where appropriate) were performed to investigate the relationship between maternal prenatal psychosocial and endocrinological stress variables. No significant correlations were found for any of the three pregnancy periods (correlation coefficients between 0.01 and 0.19 ).
Relationship between prenatal psychosocial stress and mixed-handedness. The prenatal psychosocial stress factors measured during the three periods in pregnancy were analyzed together (Table 2). No significant effects of prenatal stress on handedness in children were found for the first or second period of pregnancy. However, prenatal stress in the third period of pregnancy (wk 37-38) was associated with mixedhandedness. More daily hassles, less fear of giving birth to a handicapped child, and maternal mixed-handedness were associated with more mixed-handedness. A longer gestation was associated with a smaller chance of being mixed-handed.

Because the study group was relatively small and to have a better chance of detecting associations between prenatal stress and mixed-handedness, post hoc analyses with a stricter definition of mixed-handedness were also carried out. Mixed-handed children, defined as those who performed at least two activities with the opposite hand to that with which the child performed the remaining activities ( $11 \%$ of the children), were compared with the $75.5 \%$ of the children who performed all the activities with the same hand. The results were almost identical to those obtained with the broader definition of mixed-handedness. Again, more daily hassles in the third period of pregnancy, less fear of giving birth to a handicapped child in late pregnancy, a shorter gestation, and maternal mixed-handedness were associated with a larger chance of being mixedhanded. In addition, a trend was found for trait anxiety

Table 2. Mixed-handedness in children and maternal psychosocial prenatal stress: a logistic regression analysis with stepwise method

\begin{tabular}{|c|c|c|c|c|c|c|}
\hline & B & Wald & $p$ & OR & \multicolumn{2}{|c|}{$95 \% \mathrm{CI}$} \\
\hline Fear of a handicapped child third period & -0.55 & 9.87 & $0.002 *$ & 0.58 & 0.41 & 0.81 \\
\hline Daily hassles third period & 0.21 & 6.51 & $0.01 \dagger$ & 1.24 & 1.05 & 1.46 \\
\hline Maternal mixed-handedness & 2.02 & 5.46 & $0.02 \dagger$ & 7.53 & 1.38 & 40.93 \\
\hline
\end{tabular}

CI, confidence interval; OR, odds ratio.

The psychosocial variables of all three pregnancy periods were included in this analysis. Only the last step is presented.

$* p<0.01 ; \dagger p<0.05$; Wald's $d f=1$. 
Table 3. Maternal prenatal cortisol levels and mixed-handedness in children

\begin{tabular}{|c|c|}
\hline Predictors & $p$ \\
\hline $\begin{array}{l}\text { Mean cortisol level in the first period of } \\
\text { pregnancy }\end{array}$ & 0.75 \\
\hline $\begin{array}{l}\text { Mean cortisol level in the second period of } \\
\text { pregnancy }\end{array}$ & 0.89 \\
\hline $\begin{array}{l}\text { Mean cortisol level in the third period of } \\
\text { pregnancy }\end{array}$ & 0.51 \\
\hline $\begin{array}{l}\text { Early morning cortisol in the first period of } \\
\text { pregnancy }\end{array}$ & 0.48 \\
\hline $\begin{array}{l}\text { Early morning cortisol in the second period } \\
\text { of pregnancy }\end{array}$ & 0.48 \\
\hline $\begin{array}{l}\text { Early morning cortisol in the third period of } \\
\text { pregnancy }\end{array}$ & 0.42 \\
\hline AUC in the first period of pregnancy & 0.64 \\
\hline AUC in the second period of pregnancy & 0.95 \\
\hline AUC in the third period of pregnancy & 0.52 \\
\hline Cortisol slope in the first period of pregnancy & 0.44 \\
\hline $\begin{array}{l}\text { Cortisol slope in the second period of } \\
\text { pregnancy }\end{array}$ & 0.89 \\
\hline $\begin{array}{l}\text { Cortisol slope in the third period of } \\
\text { pregnancy }\end{array}$ & 0.51 \\
\hline
\end{tabular}

The logistic regression analysis with stepwise method, in which the cortisol variables of all three pregnancy periods were included, resulted in a nonsignificant model. The table presents the $p$ values for the cortisol variables.

measured in late pregnancy to be associated with a larger chance of being mixed-handed $(\mathrm{OR}=1.19, p=0.06)$.

Relationship between prenatal endocrinological measures and mixed-handedness. The maternal prenatal cortisol levels measured during the three periods in pregnancy were analyzed together. No significant effects of maternal cortisol on child mixed-handedness were found ( $p$ values are presented in Table 3).

\section{DISCUSSION}

In this study, we investigated whether maternal prenatal stress was associated with mixed-handedness in the offspring. Stress was assessed in three pregnancy periods by means of self-report and by measuring cortisol concentrations in saliva. Independent observations of the children's behavior were used to score the handedness of the 6-y-old offspring.

Our main finding was in the expected direction, i.e. children whose mothers had reported more daily hassles in late pregnancy had a 1.24 greater chance of being mixed-handed than did children whose mothers had reported fewer daily hassles in late pregnancy. Our post hoc analysis using a stricter definition of mixed-handedness additionally showed that mothers with greater trait anxiety had a tendency to have children with a greater chance of mixed-handedness. This supports the idea that maternal prenatal stress, measured as general stress, may influence lateralization as measured with mixed-handedness. The results are also in line with the findings of the other two recent studies $(11,12)$, which also found a relationship between general stress, instead of specific pregnancy anxiety, and mixed handedness.

However, in our study, we also found that a late pregnancyspecific pregnancy anxiety, namely, fear of bearing a handicapped child, was negatively associated with mixed- handedness $(\mathrm{OR}=0.58)$. Thus, the children whose mothers had reported more pregnancy-related anxiety had a smaller chance of being mixed-handed.

These apparently contradictory results could be explained by possible differences in late pregnancy lifestyle that go together with maternal reports of general stress and pregnancy-related anxiety in that period. Mothers reporting high levels of general stress in the form of daily hassles (and trait anxiety) would very probably be leading active, stressful lives at the end of their pregnancies. This could result in heightened activity of both the HPA axis and the sympathetic-adrenomedullary axis, with an accompanying decrease in blood flow to the uterus. Indeed, earlier research has found maternal general anxiety in late pregnancy to be related to indices of reduced uterine blood flow (27) and alterations in blood flow in the umbilical and fetal middle cerebral arteries (28). In turn, these effects could affect brain development, increasing the chance of mixedhandedness.

Conversely, mothers reporting specific pregnancy anxiety might show a different lifestyle in late pregnancy. It is very possible that women that toward the end of their pregnancy have the specific fear of having a handicapped child take good care of themselves by following a healthy diet, sleeping and resting enough daily, and avoiding hassles and stressful situations. This healthy lifestyle could explain why these women have offspring with a smaller chance of mixed-handedness. More research is needed to determine whether this hypothesis can be accepted as an explanation of how different types of prenatal maternal stress differently affect fetal development.

We did not find effects of prenatal maternal stress measured as life events and of prenatal maternal stress measured during the earlier stages of pregnancy on mixed-handedness. Therefore, the results of our study are only partly consistent with the recently published studies by Obel et al. (11) and Glover et al. (12).

There are several possible explanations for the differences in results between the studies.

First of all, the most probable reason for not finding the associations previously reported by other authors is a possible lack of power in the present study due to the relatively small sample size.

Second, the studies were carried out at dissimilar ages (6.7 y versus between 3.0 and $3.5 \mathrm{y}$ ). The possibility exists that effects of maternal prenatal stress on lateralization vary according to the developmental stage that the child is in or that the hand preference of children becomes more evident and stable with increasing age. In this latter case, the earlier results of Obel et al. and Glover $e t$ al. could be indicating that prenatal stress is related to a later stabilization of hand preference and hence to a more frequent categorization as mixed-handed at the age of 3-3.5 y. In other words, prenatal stress could be more related to a delay in the consolidation of handedness than to a stable mixed-handed pattern.

Third, different definitions were used to categorize mixedhandedness. In the present study, mixed-handedness was scored when the child performed at least one activity with the opposite hand, which is in line with the operationalization of Obel et al. (11) and with Annett's (26) definition of mixed-handedness as "a 
firm preference for different hands for different actions." Glover et al. (12) used a different definition of mixed-handedness. Mixed-handedness was scored when the mother reported two or more of these items for which the child used either hand. The differences in results may thus also have been caused by differences in defining mixed-handedness.

No relationship was found between prenatal maternal cortisol levels and offspring mixed-handedness. Earlier results in this longitudinal study showed no relationship between maternal cortisol and the offspring's temperament, behavior, and learning and memory, on the one hand (16), but a positive relationship with offspring cortisol reactivity levels on the other $(13,14)$. This could mean that these types of maternal prenatal variables are generally unrelated, as was also the case in the present study, and have independent effects on the unborn child. In fact, positive correlations between levels of cortisol and self-reported stress are often not found in stress research (29). Moreover, the associations found earlier in our study between maternal prenatal cortisol and cortisol reactivity in the offspring could at least partly be due to genetic relatedness between the mother and her child. Another possibility is that the hormone cortisol does not adequately reflect a pregnant woman's physiologic stress status. Other HPA axis hormones, such as corticotropin-releasing hormone and corticotropin, may be more important indicators of the maternal physiologic stress status.

Although we did not find clear associations between prenatal stress and mixed-handedness, we did find a negative association between gestational age and mixedhandedness. Thus, children who were older at birth were less likely to be mixed-handed. This finding is consistent with the observation that right-handedness is associated with a greater maturity at birth (26). Conversely, because all the children in our study were born after $37 \mathrm{wk}$ of gestation, this finding could be due to chance.

We also found maternal mixed-handedness to be associated with child mixed-handedness. It is known that right-handedness has a genetic component (26), but to our knowledge the genetic component has not been determined for mixed-handedness. It is interesting that Glover et al. (12) also found maternal mixedhandedness to be positively associated with the child's mixedhandedness. This points in the direction of a possible genetic component of, as well as a prenatal environmental influence on, the offspring's mixed-handedness.

The overall conclusion from this study is that in women with low to moderate levels of late pregnancy maternal stress, higher levels of daily hassles appear to be associated with more mixed handedness in their school-age offspring. Although our results were only partly consistent with those of earlier studies, the fact that the children were older and that handedness was actually observed instead of being reported by the parents can be considered methodologically strong points that make the results reliable. To gain more insight into whether the inconsistent results between the studies are due to sample sizes, type of data, operationalizations of mixed-handedness, or age, carefully designed studies are needed. Studies should be carried out in large samples, at different ages, and should include sophisticated behavioral observations for determining handedness.

Acknowledgments. The authors express their gratitude to the parents and children who participated in this study and to Anja Huizink and Pascale G. Robles de Medina for their contribution to data collection.

\section{REFERENCES}

1. Barker DJ 1995 The Welcome Foundation Lecture, 1994. The fetal origins of adult disease. Proc Biol Sci 262:37-43

2. Van den Bergh BR, Mulder EJ, Mennes M, Glover V 2005 Antenatal maternal anxiety and stress and the neurobehavioural development of the fetus and child: links and possible mechanisms. A review. Neurosci Biobehav Rev 29:237-258

3. Alonso J, Castellano MA, Rodriguez M 1991 Behavioral lateralization in rats: prenatal stress effects on sex differences. Brain Res 539:45-50

4. Alonso SJ, Navarro E, Santana C, Rodríguez M 1997 Motor lateralization, behavioral despair and dopaminergic brain asymmetry after prenatal stress. Pharmacol Biochem Behav 58:443-448

5. Weinstock M 2001 Alterations induced by gestational stress in brain morphology and behavior of the offspring. Prog Neurobiol 65:427-451

6. Satz P, Green MF 1999 Atypical handedness in schizophrenia: some methodological and theoretical issues. Schizophr Bull 25:63-78

7. Baron-Cohen S, Hammer J. 1997 Is autism an extreme form of the "male brain"? In: Rovee-Collier C, Lipsitt LP, Hayne H (eds) Advances in Infancy Research. Vol 11, Ablex Publishing, Greenwich, pp193-217

8. Annett M 1999 Left-handedness as a function of sex, maternal versus paternal inheritance, and report bias. Behav Genet 29:103-114

9. Reid HM, Norvilitis JM 2000 Evidence for anomalous lateralization across domain in ADHD children as well as adults identified with the Wender Utah rating scale. J Psychiatr Res 34:311-316

10. Orr KG, Cannon M, Gilvarry CM, Jones PB, Murray RM 1999 Schizophrenic patients and their first-degree relatives show an excess of mixed-handedness. Schizophr Res 39:167-176

11. Obel C, Hedegaard M, Henriksen TB, Secher NJ, Olsen J 2003 Psychological factors in pregnancy and mixed-handedness in the offspring. Dev Med Child Neurol 45:557-561

12. Glover V, O'Connor TG, Heron J, Golding J 2004 Antenatal maternal anxiety is linked with atypical handedness in the child. Early Hum Dev 79:107-118

13. Gutteling BM, de Weerth C, Buitelaar JK 2004 Maternal prenatal stress and 4-6 year old children's salivary cortisol concentrations pre-and post-vaccination. Stress 7:257-260

14. Gutteling BM, de Weerth C, Buitelaar JK 2005 Prenatal stress and children's cortisol reaction to the first day of school. Psychoneuroendocrinology 30:541-549

15. Gutteling BM, de Weerth C, Willemsen-Swinkels SH, Huizink AC, Mulder EJ, Visser GH, Buitelaar JK 2005 The effects of prenatal stress on temperament and problem behavior of 27-month-old toddlers. Eur Child Adolesc Psychiatry 14:41-51

16. Gutteling BM, de Weerth C, Zandbelt N, Mulder EJ, Visser GH, Buitelaar JK 2006 Does maternal prenatal stress adversely affect the child's learning and memory at age six? J Abnorm Child Psychol 34:789-798

17. Huizink AC, Robles de Medina PG, Mulder EJ, Visser GH, Buitelaar JK 2003 Stress during pregnancy is associated with developmental outcome in infancy. J Child Psychol Psychiatry 44:810-818

18. Huizink AC, Mulder EJ, Buitelaar JK 2004 Prenatal stress and risk for psychopathology. Specific effects or induction of general susceptibility. Psychol Bull 130:115-142

19. Huizink AC, Robles De Medina PG, Mulder EJ, Visser GH, Buitelaar JK 2002 Psychological measures of prenatal stress as predictors of infant temperament. J Am Acad Child Adolesc Psychiatry 41:1078-1085

20. Vingerhoets A, Jeninga AJ, Menges LJ 1989 [The measurement of daily hassles and chronic stressors: the development of the Everyday Problem Checklist (EPCL, Dutch: APL).] Gedrag Gezond 17:10-17

21. Holmes TH, Rahe RH 1967 The social readjustment rating scale. J Psychosom Res $11: 213-218$

22. Van de Willige G, Schreurs P, Tellegen B, Zwart F 1985 Het meten van 'life events': de Vragenlijst Recent Meegemaakte Gebeurtenissen (VRMG). Ned Tijdschr Psychol 40:1-19

23. Van den Bergh BR 1990 The influence of maternal emotions during pregnancy on fetal and neonatal behavior. J Prenat Perinat Psychol Health 5:119-130

24. Cohen S, Williamson GM 1987 Perceived stress in a probability sample of the United States. In: Spacapan S, Oskamp S (eds) The Social Psychology of Health. Sage Publications, Newbury Park, CA, pp 31-47

25. Spielberger CD, Gorsuch I, Leshene RE 1970 Manual for the State-Trait Anxiety Inventory. Consulting Psychologists Press, Palo Alto, CA

26. Annett M 2004 Hand preference observed in large healthy samples: Classification, norms and interpretations of increased non-right-handedness by the right shift theory. Br J Psychol 95:339-353

27. Teixeira JM, Fisk NM, Glover V 1999 Association between maternal anxiety in pregnancy and increased uterine artery resistance index: cohort based study. BMJ 318:153-157

28. Sjostrom K, Valentin L, Thelin T, Marsal K 1997 Maternal anxiety in late pregnancy and fetal hemodynamics. Eur J Obstet Gynecol Reprod Biol 74:149-155

29. Dickerson SS, Kemeny ME 2004 Acute stressors and cortisol responses: a theoretical integration and synthesis of laboratory research. Psychol Bull 130:355-391 